Preprint typeset in JHEP style. - HYPER VERSION

RUNHETC-2001-12

NSF-ITP-01-29

hep-th/0104139

\title{
Ramond-Ramond Couplings of Noncommutative D-branes
}

\author{
Hong Liu* and Jeremy Michelson ${ }^{\dagger}$ \\ New High Energy Theory Center \\ Rutgers University \\ 126 Frelinghuysen Road \\ Piscataway, NJ 08854 USA
}

\begin{abstract}
We propose the leading couplings, in an $\alpha^{\prime}$ expansion, of D-branes to Ramond-Ramond (RR) potentials in a constant NSNS $B$-field for an arbitrary choice of noncommutative parameter. The proposal is motivated by some string amplitude computations. The zero momentum couplings are topological in nature and include Elliott's formula involving the noncommutative Chern character. The finite momentum couplings are given by smearing the zero momentum operators along an open Wilson line. Comparisons between the RR couplings in different descriptions lead to a better understanding of the field redefinitions between gauge field variables (the Seiberg-Witten map) and help constrain $\alpha^{\prime}$ corrections. In particular we recover the Seiberg-Witten map conjectured by one of the authors in hep-th/0011125. We also discuss the dynamics of the transverse scalar fields and find evidence for a new derivative-driven dielectric effect.
\end{abstract}

\footnotetext{
*liu@physics.rutgers.edu

$\dagger$ jeremy@physics.rutgers.edu
} 


\section{Contents}

1. Introduction 1

2. The Wess-Zumino Action for Noncommutative D-branes

2.1 Zero momentum couplings 4

2.2 Finite momentum couplings 7

3. T-duality, Elliot formula and K-theory 8

4. Relations between different descriptions and the Seiberg-Witten Map 9

5. Derivative-driven dielectric effects 12

6. Discussions 14

\section{Introduction}

In recent years, various interesting insights have been obtained by considering D-branes in the presence of a background Neveu-Schwarz $B$-field [1, 2, 3]. While a constant $B$ field background can be gauged away in the noncompact spacetime in the closed string sector, it generates a rather nontrivial effect on the open strings on the D-branes: the low energy world-volume theory becomes noncommutative. The low energy theory on the D-branes is given by the Dirac-Born-Infeld action plus Wess-Zumino (WZ) terms. In the past, great insights have been gained into issues like gauge theory dynamics, black holes and the AdS/CFT correspondence by studying the couplings between the D-branes and bulk closed string modes. In addition studying Ramond-Ramond (RR)

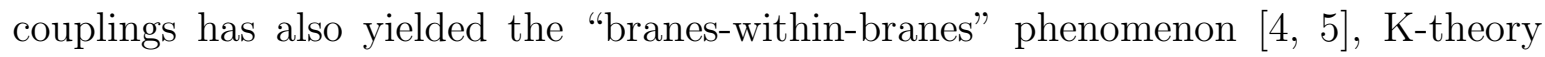
descriptions of D-branes [6, 7] and the Myers dielectric effect [8].

In this paper, we continue the study of couplings of the noncommutative D-branes to the spacetime gravity fields, following [9, 10] (see also [11, 12, 13, 14]). In [9, 10], we examined the Born-Infeld couplings to the fluctuations of the closed string metric $g_{\mu \nu}$, dilaton and the $B$-field. In this paper we will examine the couplings to RR potentials. 
As pointed out in [3], there are different descriptions of the D-brane dynamics parameterized by a noncommutative parameter $\theta$. Different descriptions are related to one another by field redefinitions which preserve the gauge orbit. The open string parameters, i.e. the metric $G$, a two-form background $\Phi$ and the coupling constant $G_{s}$, associated with each $\theta$-description can be found from the closed string background ${ }^{1}$ [3]

$$
\begin{aligned}
& \frac{1}{g+B}=\frac{1}{G+\Phi}+\frac{\theta}{2 \pi \alpha^{\prime}} \\
& G_{s}=g_{s}\left(\frac{\operatorname{det}(G+\Phi)}{\operatorname{det}(g+B)}\right)^{\frac{1}{2}}=g_{s}\left(\frac{\operatorname{det} G}{\operatorname{det} g}\right)^{\frac{1}{4}}
\end{aligned}
$$

There are three cases of (1.1) which are of particular interests:

1. $\theta=0, \Phi=B$. This is the commutative description.

2. $\Phi=0, \frac{\theta}{2 \pi \alpha^{\prime}}=-\frac{1}{g+B} B \frac{1}{g-B}$. This is the description that most naturally follows from the on-shell string amplitudes.

3. $\Phi=-B, \frac{\theta}{2 \pi \alpha^{\prime}}=\frac{1}{B}$. This is the Matrix model description [15] and is closely related background independence of noncommutative Yang-Mills theory [3], 15].

The leading order RR couplings in the commutative description are well known 16, 5, 8 They are given by the WZ terms ${ }^{2}$ plus derivative corrections which are accompanied by higher powers of $\alpha^{\prime}$

$$
S_{\mathrm{WZ}}=\mu_{p} \mathrm{~S} \operatorname{Tr} \int\left(\mathcal{P} e^{-i \iota_{\phi} \iota_{\phi}} e^{B} C\right) e^{F}+\mathcal{O}\left(\alpha^{\prime}\right) .
$$

In this paper we are interested in finding the leading order couplings (in an $\alpha^{\prime}$ expansion) in a general $\theta$-description ${ }^{3}$. Since the relations (1.1) between the open string parameters for different $\theta$-descriptions involve $\alpha^{\prime}$, the effective action in one description (e.g. with some $\theta_{1}$ ) should be considered as a resummation (along with field definitions between gauge field variables) of that in another description (e.g. with some $\theta_{2}$ ) including all higher order $\alpha^{\prime}$ corrections. For this reason, understanding the relations between different descriptions should be very useful for a deeper understanding of noncommutative gauge theories, and more generally, D-brane dynamics.

\footnotetext{
${ }^{1}$ We shall keep the closed string background $g, B$ fixed and choose either $\Phi$ or $\theta$ as a free parameter to specify the description.

${ }^{2} \mu_{p}$ is the RR charge of the brane. The pullback $\mathcal{P}$ and the contraction $\iota_{\phi}$ of the transverse scalars are defined in more detail in section 2, particularly equations (2.3) and (2.2).

${ }^{3}$ The leading action for the $\Phi=-B$ description was proposed in [14], from the connection between noncommutative D-branes and the Matrix model.
} 
We have computed various tree-level disk amplitudes for the scattering of open string gauge fields off an RR potential, and extracted the effective action to the lowest order in $\alpha^{\prime}$. The RR couplings found in this way correspond to the $\Phi=0$ description. There are several advantages to using this description. Since it follows directly from on-shell amplitudes, the low energy gauge theory dynamics is most transparent in this description. ${ }^{4}$ Also varying $B$, interpolates between the commutative description, $\Phi=B$, and the Matrix description, $\Phi=-B$. More explicitly, since (for $\Phi=0$ ), $\theta=-\left(2 \pi \alpha^{\prime}\right) \frac{1}{g+B} B \frac{1}{g-B}$, as $B \rightarrow 0, \theta \rightarrow 0$, giving the commutative description, and as $B \rightarrow \infty, \theta \rightarrow\left(2 \pi \alpha^{\prime}\right) \frac{1}{B}$, which is the Matrix model description. By contrast, in the $\Phi=-B$ description, the $B \rightarrow 0$ limit is the limit of infinite, not zero noncommutativity. Thus it is not as convenient a description of the low energy theory as the commutative gauge theory.

We shall argue that the couplings we find for $\Phi=0$ actually give the leading RR couplings for all $\Phi$ descriptions. This is partly due to the topological nature of the leading couplings of the RR potentials. The conclusion is also consistent with the known results for $\Phi=B$ and $\Phi=-B$.

Comparisons between the RR couplings in different descriptions lead to a better understanding of the field redefinitions between gauge field variables (the Seiberg-Witten map). They also constrain $\alpha^{\prime}$ corrections. In particular we shall recover the SeibergWitten map conjectured by one of the authors in [9]. We also derive some other interesting identities regarding the map.

While the amplitude calculations are in principle staightforward to carry out, the intermedate steps turn out to be quite messy and complicated. In order to not have complicated intermediate technical details obscure the simplicity of the final results, and the physics therein, we have separated this work into two parts, with this part containing the major results and physical implications, and the other containing mostly detailed calculations.

This paper is organized as follows. In section 2 we present our results from amplitude calculations. We give the RR couplings in various equivalent forms, which are useful for different purposes. In particular, we connect the commutative result (1.2) to the proposal of [14 for $\Phi=-B$. In section 3, we discuss the relations of our results in section 2 with some results in noncommutative geometry, and their consistency with T-duality. ${ }^{5}$ In section 1 we compare the RR couplings of different descriptions, from which we confirm the Seiberg-Witten map conjectured in [9] and make some remarks

\footnotetext{
${ }^{4}$ Recall that the mass shell conditions for massive open string states are defined in terms of the open string metric $G$ in the $\Phi=0$ description. For this reason, the leading order results (in terms of $\left.\alpha^{\prime}\right)$ for other values of $\Phi$ generally involve the contributions of massive open string modes.

${ }^{5}$ For the $\Phi=-B$ proposal of [14], T-duality was discussed in [17].
} 
regarding $\alpha^{\prime}$ corrections. In section 5 we focus on the transverse scalar couplings and discuss the analogue of the Myers dielectric effect [8]. We conclude in section 6 by discussing the possible relation between our discussion and anomalies in noncommutative gauge theories, and offering some speculations for the couplings for non-BPS branes.

As we were completing this work, [18, 19] appeared which has some overlap with the contents of this paper, particularly section 4 .

\section{The Wess-Zumino Action for Noncommutative D-branes}

We have extrapolated the RR couplings of a collection of noncommutative D-branes, by computing tree-level disk diagrams for the scattering of open string gauge fields off an RR potential, and extracting the effective action. The details are presented in [20].

The action found in this way corresponds to the description with $\frac{\theta}{2 \pi \alpha^{\prime}}=-\frac{1}{g+B} B \frac{1}{g-B}$. Eventually (in section 1 ) we shall argue that it applies to any $\theta$-description.

We shall present the couplings in three equivalent ways which are useful for different purposes. We will discuss their physical implications in later sections. To better understand the structure of the action, we shall first give the couplings for RR potentials at zero momentum. This captures the Lorentz tensor structure of the Yang-Mills operators. The finite momentum couplings turn out, not surprisingly, to have the form of the zero momentum operators, after smearing them along an open Wilson line [21, 22, 23, 24] following the prescription given in [9, 11].

In the following, we shall use $\hat{A}_{\mu}, \hat{\phi}^{i}$ to denote the noncommutative gauge fields and the transverse scalars. Indices longitudinal to the brane are $\mu, \nu, \cdots$ and transverse indices are $i, j, \cdots$. When appropriate we will assume that $\theta$ and $B$ have maximal rank. Then, for $p$ odd, we shall consider a Euclidean world-volume with all longitudinal directions noncommutative, while for $p$ even all longitudinal directions but time are noncommutative.

\subsection{Zero momentum couplings}

Our first expression has a close resemblance to the commutative couplings (1.2). At zero momentum, the RR potentials $C=\sum_{n} C^{(n)}$ are constant forms and the couplings of $N$ noncommutative $D p$-branes can be written as $^{6}$

$$
S_{\mathrm{WZ}}=\mu_{p} \operatorname{STr} \int\left(e^{-\iota_{\tilde{\theta}}} e^{2 \pi \alpha^{\prime} \hat{F}} \mathcal{P}\right) e^{-2 \pi \alpha^{\prime} i \iota_{[\hat{\phi}, \hat{\phi}]}} C e^{B},
$$

\footnotetext{
${ }^{6}$ Strictly speaking, the following formulas are only appropriate for topologically trivial configurations. See also footnote 11 in section 3 .
} 
where STr is the symmetrized trace over the $U(N)$ matrices, $\mu_{p}=\frac{1}{(2 \pi)^{p} g_{s} \alpha^{(p+1) / 2}}$ is the D-brane charge ( $g_{s}$ is the closed string coupling), and $\tilde{\theta}=\frac{\theta}{2 \pi \alpha^{\prime}}$. As usual, wedge products are implied in expanding the exponential and in products of forms, and the integration extracts only the $(p+1)$-form in the integrand. In evaluating products of open-string fields, the $*$-product is used.

The notation $\iota_{T}$ denotes contraction with respect to the antisymmetric tensor $T$ of rank $m$; i.e.

$$
\left(\iota_{T} \omega^{(n)}\right)_{\mu_{1} \ldots \mu_{n-m}}=\frac{1}{m !} T^{\nu_{m} \cdots \nu_{1}} \omega_{\nu_{1} \cdots \nu_{m} \mu_{1} \ldots \mu_{n-m}} .
$$

The notation $\mathcal{P}$ denotes the pullback; e.g.

$$
\mathcal{P} \omega_{\mu \nu}^{(2)}=\omega_{\mu \nu}^{(2)}+2\left(D_{[\nu} \hat{\phi}^{i}\right) \omega_{\mu] i}^{(2)}+D_{\mu} \hat{\phi}^{i} D_{\nu} \hat{\phi}^{j} \omega_{i j}^{(2)}
$$

where

$$
D_{\mu} \hat{\phi}^{i}=\partial_{\mu} \hat{\phi}^{i}-i\left[\hat{A}_{\mu}, \hat{\phi}^{i}\right]
$$

and the square brackets in (2.3) denote antisymmetrization with unit weight. Note that with a mild notational abuse,

$$
\mathcal{P}=e^{D \iota_{\hat{\phi}}}
$$

where $D \iota_{\hat{\phi}}=D_{\mu} \hat{\phi}^{i} d x^{\mu}$ is considered as a one-form in the worldvolume and a contracted vector in the transverse dimensions. That is, we can think of $D \iota_{\hat{\phi}}$ as an operator which acts on forms to the right, by contracting the vector index and antisymmetrizing the form index, thereby preserving the dimension of the form on which it acts. This reproduces (2.3).

The noncommutative field strength is

$$
\hat{F}_{\mu \nu}=\partial_{\mu} \hat{A}_{\nu}-\partial_{\nu} \hat{A}_{\mu}-i\left[\hat{A}_{\mu}, \hat{A}_{\nu}\right]
$$

and $\hat{\phi}^{i}$ are the transverse scalars. Note that the commutators in (2.4), (2.6) and in ${ }^{\iota}[\hat{\phi}, \hat{\phi}]$ in (2.1) include both the $U(N)$ and *-products. In (2.1), the symmetrized trace treats gauge covariant objects $\hat{F}, D_{\mu} \hat{\phi}^{i}$ and $\left[\hat{\phi}^{i}, \hat{\phi}^{j}\right]$ as single operators, and all products between them are $*$-products. The parentheses in (2.1) enforce that $\tilde{\theta}$ can only contract with $\hat{F}$ and/or the $D_{\mu}$ s from $\mathcal{P}$, but with nothing else. For example, with $C^{(2)}=$ $\left[\frac{1}{2} C_{\mu \nu} d x^{\mu} d x^{\nu}+C_{\mu i} d x^{\mu} d x^{i}+\frac{1}{2} C_{i j} d x^{i} d x^{j}\right]$

$$
\begin{aligned}
& \left(e^{-\iota_{\theta}} \mathcal{P}\right) C^{(2)}=\left[\frac{1}{2} C_{\mu \nu} d x^{\mu} d x^{\nu}+C_{\mu i} d x^{\mu} d x^{i}+\frac{1}{2} C_{i j} d x^{i} d x^{j}\right] \\
& +D_{\nu} \hat{\phi}^{i}\left[C_{\mu i} d x^{\mu} d x^{\nu}+C_{i j} d x^{\nu} d x^{j}\right]+D_{\mu} \hat{\phi}^{i} D_{\nu} \hat{\phi}^{j} C_{i j} d x^{\mu} d x^{\nu}-\frac{1}{2} \theta^{\tau \sigma} D_{\sigma} \hat{\phi}^{i} D_{\tau} \hat{\phi}^{j} C_{i j} .
\end{aligned}
$$


For convenience, in the following we shall set the inverse string tension $2 \pi \alpha^{\prime}=1$.

An alternative way of writing (2.1) is

$$
S_{\mathrm{WZ}}=\frac{\mu_{p}}{\operatorname{Pf}(\theta)} \mathrm{S} \operatorname{Tr} \int \star\left(e^{-i \iota_{[X, X]}} e^{B-\frac{1}{\theta}} C\right),
$$

where $\star$ is the Hodge dual ${ }^{7}$ in the noncommutative ${ }^{8}$ directions and

$$
X^{\mu}=x^{\mu}+\theta^{\mu \nu} \hat{A}_{\nu}, \quad X^{i}=\hat{\phi}^{i}\left(x^{\mu}\right), \quad\left[x^{\mu}, x^{\nu}\right]=i \theta^{\mu \nu} .
$$

Note that

$$
\left[X^{\mu}, X^{\nu}\right]=i(\theta-\theta \hat{F} \theta)^{\mu \nu}, \quad\left[X^{\mu}, X^{i}\right]=i \theta^{\mu \nu} D_{\nu} \hat{\phi}^{i}
$$

In reaching equation (2.8), and for the rest of this subsection, we need to take $\theta$ to be of maximal rank, in the sense described at the end of the introduction to this section.

While it is not obvious, the equivalence of (2.8) to (2.1) can be shown by repeatedly using the formulas

$$
\begin{gathered}
\int\left(e^{-\iota_{\theta}} \chi\right) \omega=\int \chi e^{-\iota_{\theta}} \omega, \\
e^{-\iota_{\theta}} \omega=\operatorname{Pf}(\theta) \star\left(\left(\star e^{-\theta^{-1}}\right)(\star \omega)\right),
\end{gathered}
$$

and an identity

$$
e^{D \iota_{\hat{\phi}}} e^{-\iota_{\theta}}=e^{-\iota_{\theta}} e^{\frac{1}{\theta}} e^{\iota_{\theta D \hat{\phi}}} e^{-\frac{1}{\theta}}
$$

where in (2.13) we have used the notation (2.5) and $\theta D \hat{\phi}$ is a short-hand for $\theta^{\mu \nu} D_{\nu} \hat{\phi}^{i}$.

A third way of writing (2.1) is

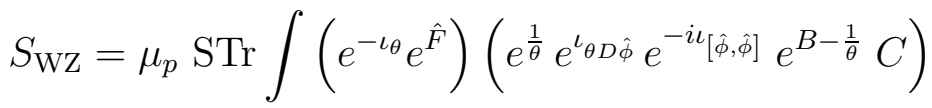

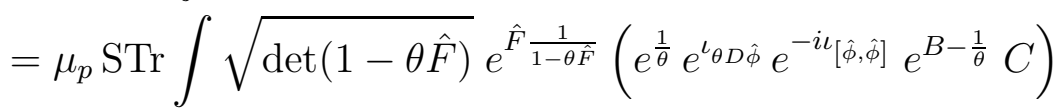

which can again be derived from (2.1) or (2.8) by using (2.11)-(2.13). In the second line above we have used an identity

$$
e^{-\iota_{\theta}} e^{\hat{F}}=\sqrt{\operatorname{det}(1-\theta \hat{F})} e^{\hat{F} \frac{1}{1-\theta \hat{F}}}
$$

\footnotetext{
${ }^{7}$ The metric dependence of the Hodge dual drops out in these equations.

${ }^{8}$ That is, for odd $p$, we take the Hodge dual in the brane, whereas for even $p$, we take the Hodge dual along the spatial directions of the brane. Thus, for odd $p$, equation (2.8) is equivalent to $S_{\mathrm{WZ}}=$ $\frac{\mu_{p}}{\operatorname{Pf}(\theta)} \operatorname{STr} \int d^{(p+1)} x\left(e^{-i \iota_{[X, X]}} e^{B-\frac{1}{\theta}} C\right)$. For even $p$, equation (2.8) can be similarly rewritten as the integral of a time-like one-form.
} 
Note that in contrast with (2.1), in (2.14) $e^{-\iota_{\theta}}$ acts only on $e^{\hat{F}}$.

Since in the above discussion, $\theta=-\frac{1}{g+B} B \frac{1}{g-B}$, if $B \ll g$ then $\theta \sim B$, and if $B \gg g$, then $\theta \sim \frac{1}{B}$. Thus the action (2.1) and/or (2.8) interpolates between (1.2) for $B=0$, to

$$
S_{\mathrm{WZ}}=\frac{\mu_{p}}{\operatorname{Pf}(\theta)} \mathrm{S} \operatorname{Tr} \int \star\left(e^{-i \iota_{[X, X]}} C\right),
$$

for $B=\infty$. Equation (2.16) is precisely the proposal of [14 based on the connection with the Matrix model. While (2.8) and (2.16) are tantalizingly close, they differ for general values of $B$ and are related by complicated field redefinitions and resummation over $\alpha^{\prime}$ corrections.

It is interesting to note that if we keep $B$ fixed, and simply take $\theta=0$ or $\theta=\frac{1}{B}^{9}$ we precisely recover (1.2) or (2.16) respectively. This observation will be important for our later claim that (2.1) and (2.8) apply to all $\theta$-descriptions in section 4 .

\subsection{Finite momentum couplings}

At finite momentum $q$, from the results in [9, 11], one expects the presence of a generalized open Wilson line $W\left(x, \mathcal{C}_{q}\right)$ 10, 12

$$
W\left(x, \mathcal{C}_{q}\right)=P_{*} \exp \left[i \int_{0}^{1} d \tau\left(q_{\mu} \theta^{\mu \nu} \hat{A}_{\nu}(x+\xi(\tau))+q_{i} \hat{\phi}^{i}(x+\xi(\tau))\right)\right] .
$$

where $q=\left(q^{\mu}, q^{i}\right)$ is the spacetime momentum of the RR potentials and the contour $\mathcal{C}_{q}$ is a straight-line $\xi(\tau)=\theta^{\mu \nu} q_{\nu} \tau$. In addition the Yang-Mills operators which appear in the zero momentum couplings (2.1), (2.8), 2.14) should now be smeared along the Wilson line (2.17) [9, 11]. This prescription, which has been checked explicitly in the couplings of noncommutative D-branes to supergravity fields in the NS-NS sector in [10, 12], is confirmed by our amplitude calculations in the RR case as well.

More explicitly, the coupling of $N$ noncommutative $D p$-branes to RR fields can be written in momentum space as ${ }^{10}$

$$
S_{\mathrm{WZ}}=\mu_{p} \int d^{10} q O_{C}(-q) C(q) e^{B},
$$

\footnotetext{
${ }^{9}$ Of course, the $\hat{A}$ and $\hat{\phi}$ in (2.1) and (2.8) are associated with $\theta=-\frac{1}{g+B} B \frac{1}{g-B}$. when taking $\theta=\frac{1}{B}$ (or 0 ) we also need to take $\hat{A}$ and $\hat{\phi}$ to be the variables assciated with $\theta=\frac{1}{B}$ (or 0 ).

${ }^{10}$ Note that in (2.18), the momentum integrations are over all directions, including the transverse components which are not conserved by the D-branes. From the D-brane point of view, this is reflected in its dependence on transverse coodinates through the factor $e^{i q \cdot \hat{\phi}}$ in the generalized Wilson line (2.17). For example, in the case $\theta \rightarrow 0$, the contour of the Wilson line (2.17) shrinks to a point and (2.17) becomes $\exp \left(i q_{i} \phi^{i}\right)$; thus integration over $q^{i}$ yields $\int d q^{i} C(q) \exp \left(i q_{i} \phi^{i}\right)=C\left(\phi(x), q^{\mu}\right)$.
} 
where $O_{C}(-q)$ is a gauge invariant operator constructed from $O_{C}(x)$, the Yang-Mills operator appearing in the integrand of (2.1) (or equivalently (2.8) and (2.14), and $W\left(x, \mathcal{C}_{q}\right)$ in 2.17$)$ via

$$
O_{C}(q)=\operatorname{Tr} \int d^{p+1} x L_{*}\left[W\left(x, \mathcal{C}_{q}\right) O_{C}(\hat{A}, \hat{\phi})\right] * e^{i q_{\mu} x^{\mu}}
$$

where the notation $L_{*}$, introduced in [9], is a short-hand for the prescription in which each single operator (i.e. one of $\hat{F}, D_{\mu} \hat{\phi}^{i},\left[\hat{\phi}^{i}, \hat{\phi}^{j}\right]$ ) contained in $O_{C}(x)$ is integrated independently over the Wilson line (2.17) using the path ordering with respect to the *-products. For example, if we had $O_{C}(x)=\hat{F}(x) \wedge \hat{F}(x)$, then we would have

$$
\begin{aligned}
O_{C}(q) & =\operatorname{Tr} \int d^{p+1} x L_{*}\left[W\left(x, \mathcal{C}_{q}\right) \hat{F}(x) \wedge \hat{F}(x)\right] * e^{i q_{\mu} x^{\mu}} \\
& =\operatorname{Tr} \int d^{p+1} x \int_{0}^{1} d \tau_{1} d \tau_{2} P_{*}\left[W\left(x, \mathcal{C}_{q}\right) \hat{F}\left(x+\xi\left(\tau_{1}\right)\right) \wedge \hat{F}\left(x+\xi\left(\tau_{2}\right)\right)\right] * e^{i q_{\mu} x^{\mu}}
\end{aligned}
$$

On expanding the Wilson line and performing the $\tau$ integrations, $O_{C}(q)$ can be written in terms of a power series of $\hat{A}$ and $\hat{\phi}$ using $n$-ary operations $*_{n}$ [25, 26]. We refer to 9] for the definition and properties of the $n$-ary operations and their the relations to the expansion of open Wilson lines. Note that the $L_{*}$-prescription symmetrizes the integrand, and so the symmetrized trace prescription in (2.1) is recovered at zero momentum.

\section{T-duality, Elliot formula and K-theory}

In this section we shall be interested in the zero momentum couplings of the gauge fields and set the transverse scalar fields to zero, in which the case the action (2.1) (or (2.14)) becomes ${ }^{11}$

$$
S_{\mathrm{WZ}}=\mu_{p} \operatorname{Tr}_{\theta}\left(e^{-\iota_{\theta}} e^{\hat{F}}\right) e^{B} C=\mu_{p} \operatorname{Tr}_{\theta} \sqrt{\operatorname{det}(1-\theta \hat{F})} e^{\hat{F} \frac{1}{1-\theta \hat{F}}} e^{B} C
$$

It may seem somewhat surprising that we have an $e^{B}$ factor in the noncommutative description, since one might expect to have only open string parameters in the action.

\footnotetext{
11 Strictly speaking, for topologically nontrivial configurations, there is not a meaningful separation of the trace $\operatorname{Tr}$ over the group indices and the spacetime integration $\int$ in a noncommutative space. Instead one simply gets a $\operatorname{Tr}_{\theta}$ which combines both, and in the trivial case becomes $\operatorname{Tr}_{\theta}=\operatorname{Tr} \int$. Another caveat is that when we take the zero momentum limit, the open Wilson line might give rise to a nontrivial normalization factor, which is also incorprated in $\operatorname{Tr}_{\theta}$. We would like to thank C. Hofman for discussions regarding these issues.
} 
The reason is the following. Suppose we compactify the theory on a torus $T^{d}$. It is well known that the scalar fields from the RR potentials transform in a chiral spinor representation of the T-duality group. However, as was argued in [27, 28], the fields which transform covariantly under the $S O(d, d ; \mathbb{Z})$ group involve not only the R-R fields $C$ but also the Neveu-Schwarz $B$-field in a combination which is precisely $D=C e^{B}$. The presence of the factor $e^{B}$ also suggests that if we had included the fluctuations of the $B$-field, it might simply enter the noncommutative description as $B \rightarrow B+\delta B$ in (2.1). It would be interesting to check whether equation (2.1) incorporates fluctuations of the $B$-field correctly. We have not computed any amplitudes with both RR and NS-NS vertex operators, and so our computation was not sensitive to fluctuations of $B$.

The RR couplings exhibit the "branes within branes" phenomenon [5], where Dbranes of lower dimensions are described by topologically nontrivial configurations of the world-volume gauge theory. In the noncommutative case, the counterparts of gauge bundles are finite projective modules of the noncommutative algebra, which can bemodulo physical processes of brane-anti brane creation and annihilation - classified by the K-theory group of the algebra. The charges of lower dimensional branes, from equation (3.1), instead of being given by the Chern character $\operatorname{ch}(E)=\operatorname{Tr}_{\theta} e^{\hat{F}}$ as in the commutative description (1.2), are given by

$$
\mu(E)=e^{-\iota_{\theta}} \operatorname{ch}(E)=\operatorname{Tr}_{\theta} e^{-\iota_{\theta}} e^{\hat{F}}=\operatorname{Tr}_{\theta} \sqrt{\operatorname{det}(1-\theta \hat{F})} e^{\hat{F} \frac{1}{1-\theta \vec{F}}}
$$

where $E$ above denotes the gauge bundle (or the projective module) on the brane.

This fits very well with the results in noncommutative geometry and the K-theoretic description of the D-branes. For a commutative manifold $\mathcal{M}$, the Chern character maps the elements of the K-theory group $K^{0}(\mathcal{M})$ of the manifold to the integral elements of the even cohomology class. However, in the noncommutative case -for concreteness

let us consider the example of a noncommutative torus $T_{\theta}^{d}$ - the Chern character $e^{\hat{F}}$, while still defining a homomorphism from $K^{0}(\mathcal{M})$ to the elements of the cohomology class, does not map to the integral elements (see e.g. [1, 29, 30]). On the other hand it was shown by Elliott [31, 32] that the K-group $K^{0}\left(T_{\theta}^{d}\right)$ can be identified with the even integral cohomology lattice of $T_{\theta}^{d}$ and in particular a K-theory class $\mu(E)$ of a module $E$ can be computed from its Chern character precisely using (3.2) (see e.g. [29, 33]). Equation (3.2) is also called the Elliott formula.

\section{Relations between different descriptions and the Seiberg- Witten Map}

In this section we shall again set the transverse scalar fields to zero and be interested in 
the couplings of the gauge fields at finite momentum, in which case the action becomes

$$
S_{\mathrm{WZ}}=\mu_{p} \int d^{p+1} k Q(k) D(-k)
$$

where $D=C e^{B}$ and

$$
\begin{aligned}
Q(k) & =\operatorname{Tr} \int d^{p+1} x L_{*}\left[e^{-\iota_{\theta}} e^{\hat{F}} W\left(x, \mathcal{C}_{k}\right)\right] * e^{i k \cdot x} \\
& =\operatorname{Tr} \int d^{p+1} x L_{*}\left[\sqrt{\operatorname{det}(1-\theta \hat{F})} e^{\hat{F} \frac{1}{1-\theta \hat{F}}} W\left(x, \mathcal{C}_{k}\right)\right] * e^{i k \cdot x} \\
& =\int d^{p+1} x Q(x) e^{i k \cdot x}
\end{aligned}
$$

In the third line we have defined $Q(x)$ as the fourier transform of $Q(k)$ to coordinate space. Note that $Q(x)$ can not be simply identified with the integrand of the first two lines since the path of the Wilson line depends on $k$.

We may expand $Q$ in terms of differential forms of different degrees, i.e.

$$
Q(k)=Q_{0}(k)+Q_{2}(k)+Q_{4}(k)+\cdots
$$

with $Q_{0}, Q_{2}, Q_{4}$ a scalar, 2-form and 4-form respectively. For example,

$$
\begin{aligned}
& Q_{0}(k)=\operatorname{Tr} \int d^{p+1} x L_{*}\left[\sqrt{\operatorname{det}(1-\theta \hat{F})} W\left(x, \mathcal{C}_{k}\right)\right] * e^{i k \cdot x} \\
& Q_{2}(k)=\operatorname{Tr} \int d^{p+1} x L_{*}\left[\sqrt{\operatorname{det}(1-\theta \hat{F})} \hat{F} \frac{1}{1-\theta \hat{F}} W\left(x, \mathcal{C}_{k}\right)\right] * e^{i k \cdot x} \\
& Q_{4}(k)=\frac{1}{2} \operatorname{Tr} \int d^{p+1} x L_{*}\left[\sqrt{\operatorname{det}(1-\theta \hat{F})}\left(\hat{F} \frac{1}{1-\theta \hat{F}}\right) \wedge\left(\hat{F} \frac{1}{1-\theta \hat{F}}\right) W\left(x, \mathcal{C}_{k}\right)\right] * e^{i k \cdot x}
\end{aligned}
$$

Then the RR couplings can be written as

$$
S_{\mathrm{WZ}}=\int\left[Q_{0} D^{(p+1)}+Q_{2} D^{(p-1)}+Q_{4} D^{(p-3)}+\cdots\right]
$$

The gauge invariance under $D \rightarrow D+d \Lambda$ requires $Q(x)$ to be closed, a statement we have only checked for some special cases. Nevertheless, here we shall assume it and explore its implications. For $Q_{0}$ to be closed it must be a constant, and it can be checked that $Q_{0}(x)=N$, which implies the following interesting identity

$$
\operatorname{Tr} \int d^{p+1} x L_{*}\left[\sqrt{\operatorname{det}(1-\theta \hat{F})} W\left(x, \mathcal{C}_{k}\right)\right] * e^{i k \cdot x}=N(2 \pi)^{p+1} \delta^{(p+1)}(k) .
$$


That $Q_{2}$ is closed implies that locally there is a one form $A$ so that $d A=Q_{2}$, i.e.

$$
(d A)(k)=\operatorname{Tr} \int d^{p+1} x L_{*}\left[\sqrt{\operatorname{det}(1-\theta \hat{F})} \hat{F} \frac{1}{1-\theta \hat{F}} W\left(x, \mathcal{C}_{k}\right)\right] * e^{i k \cdot x}
$$

For a $U(1)$ gauge group, equation (4.9) is precisely the Seiberg-Witten map conjectured in [9]. Thus the consistency of the couplings essentially confirms the conjecture of [9]. While the conjecture has a natural generalization to the $U(N)$ case, (4.9) appears to only confirm the $U(1)$ part of it.

Comparison between the RR couplings in different descriptions can give a better understanding of the Seiberg-Witten map (4.9), and also constrain $\alpha^{\prime}$ corrections. In the commutative description, it has been argued in [34] that there is no $\alpha^{\prime}$ correction for the couplings of $D^{p+1}$ and $D^{p-1}$ in (1.2). That $Q_{0}=N$ and $Q_{2}$ precisely gives the SeibergWitten map for the gauge fields, is in accord with the results of [34] and also suggests that there are no $\alpha^{\prime}$ corrections to (4.7) in the $\Phi=0$ description either, a conclusion which is otherwise hard to obtain from amplitude calculations. For the couplings of $D^{(p-3)}$, there are higher order $\alpha^{\prime}$ corrections to both descriptions; some corrections in the commutative description were found in [34] and in the $\Phi=0$ description they can be seen from the disk amplitude with two open string insertions. Thus in this case we cannot conclude that $Q_{4}=F \wedge F$ and it should be interesting to constrain the higher order corrections on both side by using the explicit Seiberg-Witten map (4.9). What we can conclude is that since $\int Q_{4}$ gives the topological charge for the $\mathrm{D}(p-4)$-brane (see section 3) in the $\Phi=0$ description and $\operatorname{Tr} \int F \wedge F$ gives the same charge in the commutative description, they must be identified, i.e. we must have

$$
Q_{4}(k=0)=\frac{1}{2} \operatorname{Tr} \int F \wedge F
$$

where $Q_{4}$ is given by (4.6). Similar statements can also be made for $Q_{6}$ and $F^{3}$ and so on.

We now know the leading order couplings to the RR potentials for all three special cases listed in the Introduction. One naturally wonders about the story for other values of $\theta$. We would like to argue that the action (2.1) and its finite momentum counterpart apply to all $\theta$-descriptions. More precisely, we claim that, if we fix the closed string background $g, B$, then for any choice of $\theta$, the leading order couplings (in terms of an $\alpha^{\prime}$ expansion) to RR potentials are given by (2.1) and its finite momentum counterpart. A heuristic argument is as follows.

1. The couplings to zero momentum RR potentials should give topological charges on the branes for any $\theta$ description. 
2. Our discussions of the topological charges in noncommutative gauge theories in section 3 and the identities (4.8), (4.10) hold for all values of $\theta$.

3. The finite momentum couplings are obtained by smearing the zero momentum couplings to an open Wilson line using the $L_{*}$ prescription (see section 2.2) $)^{12}$.

Thus from the above statements we conclude the zero momentum couplings for all $\Phi$ should be given by (3.1) and at finite momentum by (4.1) and (4.2). Another immediate consistency check is that by taking $\theta=0$ and $\theta=\frac{1}{B}$ along with a change of gauge field variables $\hat{A}$ appropriate for each $\theta$ in (2.1) we indeed recover the results for the commutative and the Matrix descriptions.

In the above we have concentrated on the part of the action involving the gauge fields. Completely parallel statements regarding the Seiberg-Witten map and $\alpha^{\prime}$ corrections can be made for the tranverse scalar fields by starting with D9-branes and doing dimensional reduction or T-duality. It would be interesting to work them out explicitly.

\section{Derivative-driven dielectric effects}

In this section we shall be interested in the dynamics of the transverse scalar fields.

When $B=0$, an important aspect of the story in the commutative description is the presence of a factor $e^{-i \iota_{\phi} \iota_{\phi}}$ in (1.2), which couples $\mathrm{D} p$-branes to RR potentials of rank higher than their dimensions(e.g. to $\left.C^{(p+3)}\right)$. While in a noncompact transverse space, it is not possible for finite number of $\mathrm{D} p$-branes to carry a net $\mathrm{D}(p+2)$ brane charge for obvious topological reasons, they can carry dipole or multipole moments, when put in a background RR field strength $F^{(p+4)}$ [8]. These dielectic effects, which turn the Chan-Paton degrees of freedom into a spacetime fuzzy geometry, require the number of branes $N>1$.

When we turn on a constant $B$ field, while we expect the above story to remain essentially the same, it is now possible to generate the dielectric effects from a single brane. The reason is that there are possible higher order $\alpha^{\prime}$ corrections like $^{13}$

$$
\int \alpha^{\prime 3} B_{\mu \nu} B_{\lambda \rho} B_{\tau \sigma}\left(\partial^{\mu} \partial^{\lambda} \phi^{i}\right)\left(\partial^{\rho} \partial^{\tau} \phi^{j}\right)\left(\partial^{\nu} \partial^{\sigma} \phi^{k}\right) F_{i j k}^{(p+4)}
$$

\footnotetext{
${ }^{12}$ Of course, we have only checked the $L_{*}$ prescription in the $\Phi=0$ description from the amplitudes. However, that it should be true for all descriptions is supported by the $\theta$ independence of the SeibergWitten map (4.9).

${ }^{13}$ We would like to thank C. Hofman for discussions regarding this point.
} 
to (1.2) when $B \neq 0$. Thus it is possible to have dielectric effects purely driven by the nontrivial profiles of transverse scalar fields in the world-volume.

In this section we would like to analyze such derivative-driven dielectric effects using the noncommutative description (2.1) and its finite momentum counterpart. The advantage is that terms like (5.1) are already present in the leading terms (2.1) in the noncommutative description, ${ }^{14}$ thus enabling a more systematic analysis. Also in terms of (2.8) a single $\mathrm{D} p$-brane with a constant $B$-field may be considered as a collection of an infinite number of D0-branes. Thus that we shall have the dielectric effects for a single brane is not surprising after all.

It is important to observe that in (2.1) in addition to the factor $e^{-i_{\hat{\phi}} \hat{\phi}^{\hat{\phi}}}$, the contraction $e^{\iota_{\theta}}$ acting on the terms in the pull-back also generate couplings to $\mathrm{RR}$ forms of higher rank. This is crucial to the absence of the coupling to $C_{i j}^{(p+3)}$ of the following form:

$$
\int_{\mathcal{B}_{p}} f(\hat{A}) \hat{\phi}^{i} \hat{\phi}^{j} C_{i j}^{(p+3)}
$$

where $C_{i j}^{(p+3)}$ should be considered as a world-volume (denoted by $\left.\mathcal{B}_{p}\right)(p+1)$-form and $f$ is fuction of $\hat{A}$. In the above equation the precise product structure, ordering or possible derivatives on $\hat{\phi}^{i}$ are not important. The presence of such a coupling would imply the possibility of generating a net $\mathrm{D}(p+2)$-brane charge. This is a very nontrivial statement, implying all terms of the form $\hat{A}^{n} \hat{\phi} \hat{\phi}$ (for some integer $n$ ) in the finite momentum version of (2.1) should cancel. We have checked the cancellations for the two lowest orders, $\hat{\phi} \hat{\phi}$ and $\hat{A} \hat{\phi} \hat{\phi}$. For example, at lowest order this is guaranteed by the following identity [35, 9]:

$$
\operatorname{Tr}\left(i\left[\hat{\phi}^{i}, \hat{\phi}^{j}\right]_{*}+\frac{1}{2} \theta^{\sigma \tau} \partial_{\sigma} X^{i} *_{2} \partial_{\tau} X^{j}\right)=0
$$

which is a special case of the recursion relation [9] (see also [36])

$$
\theta^{\mu \nu} \partial_{\nu} *_{n}\left[f_{1}, \ldots, f_{n-1}, \partial_{\mu} g\right]=i \sum_{j=1}^{n-1} *_{n-1}\left[f_{1}, \ldots, f_{j} * g-g * f_{j}, \ldots, f_{n-1}\right] .
$$

The dielectic effects appear when we look at the terms cubic in $\hat{\phi}$. For a constant RR field strength $F_{i j k}^{(p+4)}=2 f \epsilon_{i j k} \epsilon_{(p+1)}$ we find a coupling (for simplicity, we consider the $U(1)$ case)

$$
\frac{i}{3} \int\left(\frac{i}{2}\left[\hat{\phi}^{i}, \hat{\phi}^{j}\right]+\frac{1}{4} \theta^{\mu \nu}\left\{\partial_{\mu} \hat{\phi}^{i}, \partial_{\nu} \hat{\phi}^{j}\right\}\right) \hat{\phi}^{k} F_{i j k}^{(p+4)}
$$

\footnotetext{
${ }^{14}$ Recall that the effective action in one description is a resummation of that in another description including all orders in $\alpha^{\prime}$ corrections.
} 
where $\{\cdot, \cdot\}$ is an anticommutator. We shall assume that $\theta$ is large, so that we can ignore the quadratic kinetic term $\partial^{2} \phi^{i}$ from the Born-Infeld action. Combining with the $\left[\hat{\phi}^{i}, \hat{\phi}^{j}\right]^{2}$ terms in the Born-Infeld action, we get an equation of motion

$$
\left[\left[\hat{\phi}_{i}, \hat{\phi}_{j}\right], \hat{\phi}^{j}\right]=i f \epsilon_{i j k}\left(\left[\hat{\phi}^{j}, \hat{\phi}^{k}\right]-\frac{i}{2} \theta^{\mu \nu}\left\{\partial_{\mu} \hat{\phi}^{j}, \partial_{\nu} \hat{\phi}^{k}\right\}\right)
$$

All products in equations (5.5) and (5.6) are $*$-products and $i, j, \cdots$ indices are raised and lowered by the metric $g_{i j}$. All but the second term on the right hand side of the equation is simply the generalization of equations for the commutative nonabelian case to the $*$-product algebra. The new term, which arises from the contraction $\iota_{\theta}$ with the quadratic terms in the pull-back, has the intriguing stucture of a Poisson bracket with respect to $\theta$ superposed with an $S U(2)$ algebra in terms of the $*$-product. The above equations should give a derivative driven dielectric effect. It would be very interesting to analyze in more detail the solutions of (5.6). However, an exact solution appears hard to come by. We hope to return to this question in the future.

\section{Discussions}

In this paper we have investigated the RR couplings of noncommutative D-branes. We argued that the leading couplings we found for the $\Phi=0$ description apply to all $\Phi$-descriptions. The zero momentum couplings are topological, involving the Elliott formula, and thus are universal for all descriptions. The finite momentum couplings are determined partly by gauge invariance and the universality of the Seiberg-Witten map.

Our results should have a variety of applications, in addition to those we have already discussed in the paper. For example, from (3.1) and (4.1), turning the argument of [37 backwards, we may deduce the presence of chiral fermion zero modes and gain insight into e.g. the index theorem in the noncommuative geometry. For example consider a Weyl fermion on a $2 p$-dimensional noncommutative manifold with a YangMills connection $\hat{A}$. The anomalous variation of the action $\log Z(\hat{A})$ then could be given by

$$
\delta \log Z(\hat{A}) \sim \int\left[e^{-\iota_{\theta}} \operatorname{ch}(\hat{F})\right]^{(1)}
$$

which can be considered as the noncommutative generalization of the famous descent formula. Such a formula can also be postulated based on our discussion of the SeibergWitten map in section 4 (see e.g (4.10) ). 
It would be interesting to generalize the present discussion to non-BPS branes and brane-antibranes. In these cases the formula (1.2) still holds (let us for simplicity set the transverse scalars to zero); however one must replace the connection $A$ and curvature $F$ there by the so-called superconnection $\mathcal{A}$ and supercurvature $\mathcal{F}$ (see e.g. [38, 39, 40, 41]). From the results of the present paper, it is then tempting to speculate that in the noncommutative case, one simply replaces the Chern character for the superconnection by a "super-Elliott" formula

$$
\int C e^{-\iota_{\theta \tau}} e^{\hat{\mathcal{F}}}
$$

where $\tau$ takes value 1 in the non-BPS case and $\tau=\sigma_{3}$ (Pauli matrix) in the D $\bar{D}$ case, since in the $\mathrm{D} \overline{\mathrm{D}}$ case brane and anti-brane have opposite noncommutative parameter due to orientation. It would be interesting to check explicitly whether the above simple extrapolation is realized.

\section{Acknowledgments}

We are grateful for useful conversations with M. Douglas, J. Harvey, F. Larsen, L. Motl, S. Sethi, and especially C. Hofman, E. Martinec and G. Moore for important discussions. We would also like to thank C. Hofman for a careful reading of the manuscript. This work was supported by DOE grant \#DE-FG02-96ER40559. J.M. was also supported by an NSERC PDF Fellowship. J.M. thanks the ITP in Santa Barbara, where this work was completed with a little help from Grant No. PHY99-07949 of the National Science Foundation. H.L. also thanks the Chicago theory group for hospitality.

\section{References}

[1] A. Connes, M. R. Douglas and A. Schwarz, Noncommutative geometry and matrix theory: Compactification on tori, J. High Energy Phys. 02 (1998) 003; [hep-th/9711162].

[2] M. R. Douglas and C. Hull, D-branes and the Noncommutative Torus, J. High Energy Phys. 02 (1998) 008; [hep-th/9711165].

[3] N. Seiberg and E. Witten, String theory and noncommutative geometry, J. High Energy Phys. 09 (1999) 032; [hep-th/9908142].

[4] E. Witten, Small Instantons in String Theory,, Nucl. Phys. B 460 (1996) 541-559; hep-th/9511030.

[5] M. R. Douglas, Branes within Branes, in Strings, Branes and Dualities, Proceedings of the Cargèse '97 NATO ASI, (L. Baulieu, et al. ed.) Kluwer Press, 1998; [hep-th/9512077]. 
[6] R. Minasian and G. Moore, K-theory and Ramond-Ramond charge, J. High Energy Phys. 11 (1997) 002; [hep-th/9710230].

[7] E. Witten, D-Branes and K-Theory, J. High Energy Phys. 12 (1998) 019; [hep-th/9810188].

[8] R. C. Myers, Dielectric Branes, J. High Energy Phys. 12 (1999) 022; hhep-th/9910053].

[9] H. Liu, *-Trek II: $*_{n}$ Operations, Open Wilson Lines and the Seiberg-Witten Map, hep-th/0011125.

[10] H. Liu and J. Michelson, Supergravity Couplings of Noncommutative D-branes,, hep-th/0101016.

[11] S. R. Das and S. P. Trivedi, Supergravity Couplings to Noncommutative Branes, Open Wilson Lines and Generalised Star Products,, J. High Energy Phys. 02 (2001) 046; hep-th/0011131].

[12] Y. Okawa and H. Ooguri, How Noncommutative Gauge Theories Couple to Gravity, Nucl. Phys. B 599 (2001) 55-82; [hep-th/0012218].

[13] Y. Okawa and H. Ooguri, Energy-momentum Tensors in Matrix Theory and in Noncommutative Gauge Theories, CALT-68-2319, CITUSC/01-003, hep-th/0103124.

[14] S. Mukhi and N. V. Suryanarayana, Chern-Simons Terms on Noncommutative Branes, J. High Energy Phys. 11 (2000) 006, [hep-th/0009101];

Talk by N. V. Suryanarayana at Strings 2001, Mumbai, India; http://theory.theory.tifr.res.in/strings/Proceedings/nemani.

[15] N. Seiberg, A Note on Background Independence in Noncommutative Gauge Theories, Matrix Model and Tachyon Condensation, J. High Energy Phys. 09 (2000) 003; [hep-th/0008013].

[16] M. Li, Boundary States of D-Branes and Dy-Strings, Nucl. Phys. B 460 (1996) 351-361; [hep-th/9510161].

[17] R. Tatar, T-duality and Actions for Non-Commutative D-Branes,, HU-EP-00/51, hep-th/0011057.

[18] Y. Okawa and H. Ooguri, An Exact Solution to Seiberg-Witten Equation of Noncommutative Gauge Theory, CALT-68-2325, CITUSC/01-010, NSF-ITP-01-25, hep-th/0104036.

[19] S. Mukhi and N. V. Suryanarayana, Gauge-Invariant Couplings of Noncommutative Branes to Ramond-Ramond Backgrounds, hep-th/0104045.

[20] H. Liu and J. Michelson, *-Trek III: The Search for Wess-Zumino, in preparation. 
[21] N. Ishibashi, S. Iso, H. Kawai, Y. Kitazawa, Wilson Loops in Noncommutative Yang Mills, Nucl. Phys. B 573 (2000) 573-593; [hep-th/9910004].

[22] J. Ambjorn, Y. M. Makeenko, J. Nishimura and R.J. Szabo, Lattice Gauge Fields and Discrete Noncommutative Yang-Mills Theory, J. High Energy Phys. 05 (2000) 023; [hep-th/0004147].

[23] S. R. Das and S.-J. Rey, Open Wilson Lines in Noncommutative Gauge Theory and Tomography of Holographic Dual Supergravity, Nucl. Phys. B 590 (2000) 453-470, [hep-th/0008042];

S. Rey and R. von Unge, S-duality, noncritical open string and noncommutative gauge theory, Phys. Lett. B 499 (2001) 215, [hep-th/0007089].

[24] D. J. Gross, A. Hashimoto and N. Itzhaki, Observables of Non-Commutative Gauge Theories, NSF-ITP-00-94, hep-th/0008075.

[25] M. R. Garousi, Non-commutative world-volume interactions on D-brane and Dirac-BornInfled action, Nucl. Phys. B 579 (2000) 209-228; [hep-th/9909214].

[26] H. Liu and J. Michelson, *-TREK: The One-Loop N=4 Noncommutative SYM Action, hep-th/0008205.

[27] D. Brace, B. Morariu and B. Zumino, T-Duality and Ramond-Ramond Backgrounds in the Matrix Model, Nucl. Phys. B 549 (1999) 181-193; [hep-th/9811213].

[28] M. Fukuma, T. Oota and H. Tanaka, Comments on T-dualities of Ramond-Ramond Potentials, Prog. Theor. Phys. 103 (2000) 425-446; hep-th/9907132].

[29] A. Schwarz, Morita equivalence and duality, Nucl. Phys. B $\mathbf{5 3 4}$ (1998) $720-$ 738; thep-th/9805034].

[30] C. Hofman and E. Verlinde, Gauge Bundles and Born-Infeld on the Noncommutative Torus, Nucl. Phys. B 547 (1999) 157-178; hep-th/9810219.

[31] G. A. Elliott, On the K-theory of the $C^{*}$-algebra generated by a projective representation of a torsion-free discrete abelian group, in Operator Algebras and Group Representations, Vol 1. 1984 Pitman, London.

[32] M. Rieffel, Projective modules over higher-dimensional noncommutative tori, Canad. J. Math. 40 (1988) 257-338.

[33] A. Konechny and A. Schwarz, Introduction to M(atrix) theory and noncommutative geometry, hep-th/0012145.

[34] N. Wyllard, Derivative corrections to D-brane actions with constant background fields, Nucl. Phys. B 598 (2001) 247-275; [hep-th/0008125]. 
[35] T. Mehen and M. B. Wise, Generalized $\star-$ Products, Wilson Lines and the Solution of the Seiberg-Witten Equations, J. High Energy Phys. 12 (2000) 008; [hep-th/0010204].

[36] Y. Kiem, D. H. Park and S. Lee, Factorization and generalized *-products, hep-th/0011233.

[37] M. Green, J. A. Harvey, G. Moore, I-Brane Inflow and Anomalous Couplings on D-Branes, Class. and Quant. Grav. 14 (1997) 47-52; [hep-th/9605033].

[38] C. Kennedy and A. Wilkins, Ramond-Ramond Couplings on Brane-Antibrane Systems,, Phys. Lett. B 464 (1999) 206-212; [hep-th/9905195].

[39] P. Kraus and F. Larsen, Boundary String Field Theory of the DDbar System, hep-th/0012198.

[40] T. Takayanagi, S. Terashima and T. Uesugi, Brane-Antibrane Action from Boundary String Field Theory, J. High Energy Phys. 03 (2001) 019; [hep-th/0012210].

[41] J. H. Schwarz and E. Witten, Anomaly Analysis of Brane-Antibrane Systems, J. High Energy Phys. 03 (2001) 032; hep-th/0103099]. 